\title{
CRISIS Y RESILIENCIA DEL ESTADO EN AMÉRICA LATINA UNA PROPUESTA DE REFLEXIÓN A PARTIR DE LA CONFLICTIVIDAD SOCIAL
}

\author{
CRISIS AND STATE RESILIENCE IN LATIN AMERICA \\ A REFLECTION APPROACH FROM SOCIAL CONFLICTIVITY
}

\author{
Victoria Inés Darling ${ }^{(*)}$ \\ Universidad Autónoma de México, Ciudad de México, México.
}

\begin{abstract}
Resumen: A partir de un diagnóstico que considera tres procesos de crisis sociopolíticas a inicio del presente siglo, el artículo aborda la problemática de la resiliencia del Estado en América Latina. En el marco del incipiente agotamiento del ciclo progresista, se recupera teóricamente el pensamiento de René Zavaleta con el fin de profundizar la interpretación de las crisis y sus consecuencias. Se abordan la memoria en tanto estructura hegemónica, los momentos constitutivos y la paradoja señorial en tanto cristalizaciones conceptuales que redundan en una epistemología crítica, atribuyendo especificidad al derrotero del Estado latinoamericano.
\end{abstract}

Palabras clave: Estado; Movimientos sociales; Crisis; Zavaleta; América Latina.

Abstract: Starting with a diagnosis that considers three processes of sociopolitical crises that occurred at the beginning of this century, the article leads the issue of the resilience of the State in Latin-America. Under the progressive depletion of the progress president cycle, the thought of René Zavaleta is recovered theoretically in order to deepen the understanding of the crisis and its consequences. Memory as hegemonic structure, constitutive moments and "lordly paradox" are concepts to be worked in order to contribute in a critical epistemology so as to consider and attribute specificity to the State course in Latin American state.

Key words: State; Social movements; Crises; Zavaleta; Latin-America.

(*) Doctora en Ciencias Políticas y Sociales por la Universidad Nacional Autónoma de México. Profesora de la Universidade Federal da Integração Latino-americana. E-mail: <darling.victoria@gmail.com> 


\section{INTRODUCCIÓN}

Las ideas que presentamos tienen por objetivo ahondar en algunos conceptos teóricos que responden inquietudes generales del pensamiento crítico latinoamericano y que su vez, adquieren nitidez en el análisis de situaciones conflictivas concretas del presente. Para darles cuerpo y contundencia, recuperamos una primera propuesta argumental en forma de diagnóstico seguido de preguntas, de modo de poder ir deshilvanando a través de ellas la suma de conceptos analíticos que entendemos, conforman un andamiaje teórico complejo del que sólo podemos recuperar una parte. El esquema teórico recuperado para al análisis se construye a partir de la consideración de diferentes obras del boliviano René Zavaleta, de sus elaboraciones, conceptos explicativos y críticas.

Lo cierto es que a lo largo del ciclo de movilizaciones que se desata en América Latina en el año 2000 ocurren crisis sistémicas que en algunos casos culminan con la renuncia o huida de los Presidentes de turno. Esto ocurrió en Bolivia en 2003, en Ecuador en 2000 y 2005, y en Argentina hacia finales del año 2001. Se trata de episodios en aquel entonces novedosos porque ocurrieron en regímenes democráticos con plena vigencia de derechos, e incluso, como se ha constatado, la caída del primer mandatario no significó la anulación del régimen, sino que el resto de las instituciones continuaron funcionando, en espera del recambio presidencial. La peculiaridad de la caída de Presidentes en el siglo XXI en América Latina, radica en que en esta oportunidad la presión fue ejercida por sectores populares que organizados en movimientos sociales se articularon y ocuparon el espacio público para manifestar sus demandas. Las movilizaciones que dieron lugar a crisis de magnitud fueron la catalización de un proceso prolongado de cuestionamiento directo a la implantación de políticas neoliberales que trajeron como resultado altos índices de desempleo, precarización de la educación pública, distanciamiento de lo político como asunto común, y erosión de los lazos colectivos, entre tantos otros.

La propuesta del presente trabajo es cuestionar la resiliencia del Estado en tiempos de crisis acorde al ciclo de protestas que va del 2000 al 2006 en América Latina, de modo de recuperar dicho proceso crítico de aprendizaje para el análisis de la trayectoria estatal que se desprende del 2006 hasta nuestros días, tiempo en que asoma la llamada "crisis del relato progresista".

Es posible considerar que los gobiernos de Argentina, Ecuador, Bolivia, Brasil, Uruguay y Venezuela se encuentran en una intensa crisis de liderazgo político, sobre todo si consideramos válida la hipótesis de un lento agotamiento de las ideas que orientaron la preferencia por políticas tendientes a consolidar la soberanía nacional, la promoción industrial, el cuidado de los bienes comunes naturales, la inclusión social y la lucha contra la desigualdad en el marco de una crítica al paradigma paradigma esencialmente neoliberal. Alertando al respecto de profecías autocumplidas, no se pretende colaborar en dicha crisis, sino profundizar en el análisis de sus razones de modo de interpretar sus condicionantes.

A partir de la consideración de las crisis como espacios de significación colectiva que alberga y vela contradicciones de clase, proponemos una lectura que actualice el debate en el contexto de situaciones políticas concretas. Con estas consideraciones pre- 
tenderemos alcanzar una revigorización teórica que apuntale su estudio en términos de Estado ampliado de modo de alcanzar una mejor comprensión de las formas posibles de alcanzar su transformación, o bien, la posibilidad de una construcción política alternativa.

\section{EL DEVELAMIENTO DE LA REALIDAD}

Aún cuando las sucesivas movilizaciones ocurridas en el inicio de siglo cuestionaron la forma de representación vigente, reclamaron autonomía y se apropiaron de sus territorios - en el campo y en la ciudad - de manera renovada, aún cuando en toda la región el ciclo de protestas incorporó a movimientos campesinos, indígenas, Sin tierra, desocupados, universitarios, sindicatos, sectores urbanos empobrecidos, ambientalistas e incluso organizaciones de mujeres, no ocurrió siquiera un atisbo de poder dual, en tanto instancia o "grieta" en el seno del propio Estado que, debilitado por una rebelión de "abajo", perdiera su capacidad para reclamar y obtener obediencia al calor de la conformación de un antagonismo de poder real. Debido a ello nos permitimos preguntar: ¿Qué elementos conducen a la reproducción de las formas políticas conocidas en desmedro del ensayo de formas alternativas?

En Argentina, la crisis del año 2001 condujo a la retención del dinero de los ahorristas en los bancos, a la creación de formas alternativas de empleo, a la ocupación de fábricas que habiendo entrado en bancarrota fueron recuperadas por sus trabajadores, se crearon clubes de trueque que con créditos reemplazaron el papel moneda, se crearon monedas paralelas al peso (como el patacón, y el lecop, entre otras) se llevaron adelante asambleas barriales que en los parques públicos de la ciudad de Buenos Aires, que definían los problemas y preocupaciones más importantes de los vecinos. En ese contexto de crisis, donde el rechazo a la clase política era expreso, manifestándose en protestas y marchas que ocasionaron además de 39 muertos, la renuncia de cinco presidentes en doce días, la salida escogida fue la reconstrucción del Estado. En un contexto donde la economía como tal no ofrecía respuestas, donde la auto organización parecía el camino para realizar política de manera honesta y desvinculada, se optó por el camino conocido: revivir el Estado y desarticular las experiencias de auto organización de la sociedad civil. Se eligió a un ex vicepresidente (de Carlos Menem), conocido por su accionar político en la provincia de Buenos Aires, esposo de una diputada nacional que se volvió reconocida por su labor de base, que obtuvo fuertes críticas por su acción paternalista y clientelar.

En Ecuador ocurrió algo similar, hacia fines de 2004 el entonces presidente Lucio Gutiérrez tomó la decisión de modificar los miembros de la Corte Suprema de Justicia generando la sospecha de que próximos a ser nombrados serían de su confianza. Esto disparó un clima de descontento que fue incrementándose. Incluso, la anulación de las investigaciones contra ex presidentes, como Abdalá Bucaram, acusados de malversación de fondos provocó mayor indignación. El clima de tensión se completaba con la crisis económica y la extensión de la pobreza. Los primeros meses de 2005 las protestas fueron diarias y se centraron en Quito. Tuvieron como protagonistas a sectores medios, agrupaciones de jóvenes y miembros de diversos colectivos indígenas (la CONAIE había apoyado la candidatura de Gutiérrez, luego, a los ocho meses de gobierno, con el acuer- 
do Gutiérrez-gobierno de Estados Unidos, la CONAIE manifestó su distanciamiento). Se crearon: ollas populares, encuentros musicales en las calles, reuniones en las plazas, debates en radios contestatarias. Una frase que evocaba la experiencia de diciembre de 2001 en Argentina sintetizaba el reclamo: "[...]qué se vayan todos!" El movimiento de los "forajidos" se construyó por iniciativas espontáneas. Las universidades, la radio comunitaria y el debate en la calle expresaban el rechazo a los partidos políticos o agrupaciones con líderes. El autodenominado "pueblo", recuperó el sentido de la política y con ella la definición del sentido de la auto-representación.

¿Acaso estas movilizaciones generaron un cambio en el sistema político? ¿Lograron "que se fueran todos" renovando la praxis política?

En Bolivia, la situación del 2003 es conocida y remite a la guerra del gas como detonante de un ciclo de protestas de mayor duración, iniciado en Cochabamba en el 2000 con la guerra del agua. En octubre no dejó de escucharse por las calles del Alto "Fuera Goni vendepatria!". A principios de 2003 el gobierno anunció medidas económicas restrictivas debido al déficit fiscal y de acuerdo a un tratado firmado con el Fondo Monetario Internacional. En esta oportunidad se decretó un impuesto al salario afectando con esta medida principalmente a los trabajadores públicos. El caso de los policías fue el más resonante porque su protesta llegó a la Plaza Murillo donde había fuerzas militares a las que se le dio la orden de "descomprimir" el conflicto. El enfrentamiento arrojó veinte muertos y en virtud de las críticas, el gobierno debió echar atrás la medida retardando su propia crisis final. Tiempo después, hacia octubre, ante rumores que descubrían la intención gubernamental de exportar gas boliviano por puertos chilenos a la costa oeste de los EEUU, México y Chile, comenzaron a desplegarse movilizaciones en la ciudad de La Paz teniendo como eje la ciudad de El Alto. La crónica indica que "Cerca de 20 mil personas, entre trabajadores, cocaleros, campesinos y estudiantes, convocados por el Movimiento Al Socialismo (MAS), el Estado Mayor del Pueblo (EMP) y la Central Obrera Boliviana (COB) se movilizan por el centro de las ciudades de La Paz, El Alto, Cochabamba y Santa Cruz en repudio a la exportación del gas y a la firma del ALCA. El conjunto de organizaciones que participan de la medida coincide en que el gas debe ser industrializado y utilizado en el país" (OSAL, 2003, p. 149). La experiencia popular demostró la novedad de un movimiento territorial de base urbana, formado por jóvenes de escasos recursos y en su mayoría trabajadores precarios de la periferia de La Paz que afirmaron su identidad indígena. El reclamo se volvió masivo y el pasar de los días generó un clima de enardecimiento que sumó a la clase media, principalmente universitarios, maestros rurales y urbanos, también trabajadores administrativos.

La creciente represión no generó una merma en los episodios de protesta sino que por el contrario, agudizó la crisis. Hacia el 15 de octubre de 2003 el presidente anunció en conjunto con los partidos políticos que conformaban la coalición gobernante, el lanzamiento de una consulta popular para saber la opinión de los bolivianos en relación a la exportación del gas, asimismo proponía la incorporación de la figura de la Asamblea Constituyente al texto constitucional así como la revisión de la Ley de Hidrocarburos. Como respuesta, los líderes de la oposición, Evo Morales y Felipe Quispe, realizaron un llamamiento a no aceptar la convocatoria gubernamental y solicitan una vez más, 
la renuncia del Primer Mandatario. Al día siguiente, mineros, cocaleros y las organizaciones que conforman la Federación de Juntas Vecinales de El Alto se movilizaron exigiendo la renuncia de Sánchez de Lozada. Las movilizaciones comenzaron a reiterarse, además de la nacionalización, se llamaba a una Asamblea Constituyente en Bolivia.

¿Acaso fueron consideradas formas de ejercicio del poder alternativo, centrada en las juntas vecinales del Alto? ¿Fueron analizadas iniciativas de asambleas comunitarias?

Lo cierto es que tanto en Argentina, como en Bolivia y Ecuador, cada una de las crisis desatadas culminó con un nuevo llamado a elecciones para, partiendo de la competencia prestablecida por constituciones liberales, votar por candidatos aptos según los preceptos de la democracia representativa. En ese sentido, nos preguntamos ¿Por qué el Estado es tan atractivo? ¿Qué sucede cuando pareciera que las condiciones para crearlo todo de nuevo están dadas y esto no se cristaliza? ¿Cómo no pensar en nuevas formas de organización política en territorios que manifiestan su reclamo de autonomía y que gritan con hartazgo "qué se vayan todos"? ¿Cómo no vincular la fortaleza de quienes destituyen con la debilidad de lo que representan quienes son destituidos? ¿Cómo explicar la resiliencia de la forma estatal en América Latina?

\section{LA MEMORIA}

Existen algunos elementos que pueden ser de utilidad para indagar en la seducción de la forma estatal. Uno de ellos, guardado en una suma precepciones, recuerdos y deseos que configuran la memoria, es la construcción del propio Estado en cada uno de nuestros países, y en dicha trayectoria, la referencia a los momentos en que el Estado fue hegemónico y alcanzó su mayor nivel de imbricación con la sociedad civil.

Suele decirse que el pasado está contenido en el presente, sin embargo, existen circunstancias en que el pasado contiene de manera tan encorsetada al presente, que permite un espacio muy reducido para crear realidades nuevas.

Cuando en sucesivas entrevistas se consultó a quienes se movilizaron en las crisis de 2001 en Argentina, 2003 en Bolivia y 2005 en Ecuador por aquel pasado que consideraban "su mejor momento político vivido", las referencias reiteradas estuvieron fuertemente vinculadas a los gobiernos nacional-populares.

En Bolivia, se comentaba que aquel tiempo en que "no faltaba nada", en que se tenía "orgullo de ser boliviano" era el tiempo donde había "hornos de fundición". Pues, la mención de los hornos remite al tiempo en que se consideraba que los minerales extraídos de las minas podrían ser, a través de un complejo procesamiento, industrializados. Minas, recursos y guerra son conceptos que se entrecruzan configurando percepciones. Y resultan numerosas las referencias que se desprenden de la apropiación de recursos, no sólo a nivel interno sino también en relación a potencias extranjeras que en diversos puntos de la historia desmembraron el país sembrando una intensa discordia aún presente de la memoria larga de los bolivianos. Como sabemos, hay dos momentos históricos que marcan la realidad boliviana. Uno es la guerra del Chaco, donde la evocación a las batallas revela un momento e intersubjetividad crucial. Y en segundo lugar, 
la revolución de 1952, momento en que la sociedad boliviana es liderada por la clase obrera alcanzando uno de los mayores grados de autodeterminación. Las minas, soporte económico defendido por sus trabajadores, fueron nacionalizadas en dicha oportunidad. Esta fue una victoria popular a la que se llegó por medio de luchas concatenadas. La reforma agraria y el voto universal fueron también parte del "triunfo" de la revolución.

En el caso de Ecuador, los vestigios que la memoria de los procesos dominantes recupera se asocian a la fuerte presencia de los militares. Referencias a "los beneficios que generan para los sectores más humildes", para "el afianzamiento de las libertades políticas" y a la "custodia de la democracia", se encuentran en muchos de los testimonios de los ecuatorianos entrevistados en Quito. La presencia de militares nacionalistas en Ecuador genera una adhesión que sólo puede explicarse comprendiendo la transformación que la sociedad ecuatoriana tuvo de la mano de José María Velasco Ibarra a mediados de la década de los '40. En términos de aspiraciones populares, el gobierno militar asumió un periodo de transición a la democracia que, plagado de ambivalencias, encausó una oleada anti-oligárquica. La figura de Velasco logró encausar esperanzas de sectores sociales emergentes y excluidos tanto en la costa como en la sierra. Aún cuando su presencia no creó bases institucionales, un proceso de industrialización o acuerdos de larga data histórica, como los casos de Perón en Argentina o Vargas en Brasil, su presencia es insoslayable de las evocaciones sobre aquel pasado mejor. Velasco realizó la "[...]prolongación exitosa y estable hacia el Estado de los mecanismos clientelares y paternalistas surgidos en las haciendas serranas [...] creando un Estado transformista[...]" (OSPINA, 2005, p. 83), en ese sentido, desmanteló la complejidad de las relaciones de poder oligárquicas creando un Estado moderno basado en relaciones político-personales propias. El liderazgo carismático militar continúa teniendo desde mediados de siglo características tentadoras que se reproducen y evolucionan en nuevas características. El autoritarismo encuentra allí también cabida ya que prácticas populares y mando militar se mixturan. En ese sentido, la presidencia de Lucio Gutiérrez comprendió la situación y desarrolló en condición de posibilidad un intento de imitación de aquellos modos de hacer política (también en términos de sostenimiento de redes clientelares para el involucramiento de los sectores populares). Un Presidente nacionalista militar evocaba a otro Presidente nacionalista militar.

Ahora bien, las instituciones tradicionales se ponen en evidencia una vez más en torno de argumentos que refieren a tiempos en que "los pobres eran tenidos en cuenta" en Ecuador. Otras referencias, más cercanas a la afectividad popular, evocan a "las comunidades". Las comunidades a diferencia de la primera impresión que resulta de la escucha, no remiten a colectivos indígenas sino a espacios horizontales que la Iglesia Católica colaboró en recrear.

La sociedad ecuatoriana, identificada como una nación profundamente religiosa, ha experimentado en las últimas décadas un proceso continuo de secularización, que se expresa de diferentes formas. Ser ecuatoriano se identificaba con católico, pero hoy esa identidad ha cambiado, va cambiando sustancialmente; las transformaciones sociales, con sus efectos racionales y científicos, han fragmentado la visión del mundo proporcionada por el cristianismo y descentralizaron lo institucionalizado (ritos, símbolos, mitos, instituciones) (RODAS, 2009, p. 299). 
La década de los años 60 en particular estuvo plagada de intentos de reconocer en las poblaciones desfavorecidas, claramente indígenas, un sujeto revolucionario. Monseñor Leónidas Proaño, Obispo de Riobamba a mediados de los años 50, fue uno de los impulsores de esta corriente. La idea de "una teología que partiera de la palabra viva de la realidad de nuestros pueblos", trabajó identificando al indígena como el más pobre de los pobres.

En Argentina, la referencia nacional y popular sigue siendo la lente desde la cual se miran los procesos políticos en Argentina.

Nosotros creemos que hasta ahora ningún gobierno ha sido para o de los trabajadores, ni el peronismo. Creemos en algo nuevo. Ahora no tenemos relación con el gobierno, depende de la presión, pero es oposición. Es cierto que el MTD Evita, o D'Elia tiene beneficios que el resto no tiene pero eso no replantea una alianza (Información verbal) ${ }^{(1)}$.

El populismo encarnado en el gobierno de Juan Domingo Perón supuso un proceso homogeneizador en términos sociales que dio dimensión nacional a los derechos. Aquellas conquistas como el sufragio femenino, el trabajo masivo, la salud como garantía, y la elevación del nivel educativo, se universalizaron. Asimismo, la expansión política incorporó el proceso de asalarización masiva habilitando la creación de organizaciones laborales sólidas que involucraban a grandes masas de obreros al campo político. La mediación entre capital y trabajo fue la bandera del gobierno y una efectiva estrategia de articulación que consiguió convertir sus prácticas en hegemónicas. Perón representó los intereses de un sector nacionalista que planteó la necesidad de crear un desarrollo industrial independiente que incorporara a los trabajadores al sistema político-económico. Incluso, desde la Secretaría de Trabajo se sancionaron leyes que establecieron un salario mínimo que procurara abastecer dignamente las necesidades de alimentación y vivienda. Se estableció el seguro social y la jubilación como derecho, se crearon los llamados "tribunales de trabajo", cuyo objetivo era mediar en los conflictos con el Estado. En suma, las mejoras salariales, el posicionamiento preferencial de las organizaciones sindicales, una renovada política social y el reconocimiento de las asociaciones de profesionales generaron una aceptación creciente que vieron en la generación de mecanismos de movilidad ascendente, mejores oportunidades de vida.

Sin embargo, la lenta descomposición del populismo hacia mediados de los años '50 como resultado de la crisis de la dominación que se materializó en el golpe de Estado contra Perón, supuso la paulatina fragmentación de las solidaridades sociales previamente gestadas. Esto configuraría una marca en la memoria al momento de referirse a "aquellos tiempos mejores". Después del peronismo, "ni la pérdida de homogeneidad territorial, ni los grandes actores corporativos en crisis, siguieron estructurando identidades estables" (ABOY CARLÉS, 2009, p. 80).

(1) Información ofrecida por Gleyza, entrevista con identidad preservada octubre de 2010. 


\section{LA ESTRUCTURA HEGEMÓNICA}

Analizando cada caso, la crisis trae al presente la recuperación de experiencias que en la actualidad adquieren sentido y nueva vitalidad. Aquello que se rememora como "el mejor momento vivido" contiene un sentido voluntario de deseo porque vuelva a suceder, más aún, permea las aspiraciones y la creación de proyectos políticos.

Y aquí resulta fundamental recuperar aquello que Marx (1852) afirmaba en el 18 brumario de Luis Bonaparte,

[...] los hombres hacen su propia historia, pero no la hacen a su libre arbitrio, bajo circunstancias elegidas por ellos mismos, sino bajo aquellas circunstancias con que se encuentran directamente, que existen y les han sido legadas por el pasado. La tradición de todas las generaciones muertas oprime como una pesadilla el cerebro de los vivos. Y cuando éstos aparentan dedicarse precisamente a transformarse y a transformar las cosas, a crear algo nunca visto, en estas épocas de crisis revolucionaria es precisamente cuando conjuran temerosos en su exilio los espíritus del pasado, toman prestados sus nombres, sus consignas de guerra, su ropaje, para, con este disfraz de vejez venerable y este lenguaje prestado, representar la nueva escena de la historia universal (MARX, 2015 [1852], p. 4).

A este proceso de recuperar un pasado que encorseta, que reduce las posibilidades a las conocidas Gramsci lo denomina "estructura hegemónica", una dinamización progresiva de las estructuras del Estado hegemónico, entendiéndolo como Estado ampliado, al que le es inmanente lo social. De aquí la necesidad de poner en crisis la estructura hegemónica de la que forman parte los sujetos de confrontación si aquello que se procura es romper con el sentido común que la legitima.

La revolución social [...] no puede sacar su poesía del pasado, sino solamente del porvenir. No puede comenzar su propia tarea antes de despojarse de toda veneración supersticiosa por el pasado. Las anteriores revoluciones necesitaban remontarse a los recuerdos de la historia universal para aturdirse acerca de su propio contenido. La revolución [...] debe dejar que los muertos entierren a sus muertos, para cobrar conciencia de su propio contenido (MARX, 2015 [1852], p. 5).

Esta aseveración permite comprender la certeza de que "sólo puede desearse aquello que se conoce" y en ese sentido, sólo parece posible construir aquello que alguna huella amable dejó en la memoria colectiva.

\section{LAS CRISIS}

Una de las contribuciones más ricas de los variados análisis de René Zavaleta remite a su obra "El revolución de 1952 y las tendencias sociológicas emergentes", de 1974. Allí Zavaleta analiza en detalle la matriz boliviana de 1952, es decir, la estructura social y las tendencias políticas críticas al Estado oligárquico, de la mano del Movimiento Nacionalista Revolucionario y el proletariado minero en conformación. 
De aquí se derivan dos elaboraciones teóricas que es posible aprehender como herramienta analítica para realidades que exceden la boliviana. La primera de ellas es la categoría de crisis en tanto "escuela". La segunda consiste en la necesidad de que una clase social se conozca a sí misma, y por consiguiente, pueda alcanzar un horizonte de visibilidad sobre la realidad de la que es parte.

Zavaleta considera que la crisis es un momento creativo por naturaleza. Una situación privilegiada para observar la desnudez de los acontecimientos y en ellos, los roles que los diferentes actores intervinientes juegan.

Es razonable concebir la crisis como un instante anómalo en la vida de una sociedad, y eso querría decir una hora en la que las cosas no se presentan como son en lo cotidiano y se presentan en cambio como son en verdad. [...]. La crisis actúa no como una forma de violencia sobre el orden de la rutina, sino como una aparición patética de las puntas de la sociedad que, de otra manera, se mantendrían sumergidas y gelatinosas (ZAVALETA, 2008 [1986], p. 19).

Desde este punto de vista la crisis puede ser también un "método de conocimiento". A partir de los síntomas de descomposición de las formas políticas se puede a rastrear el momento de articulación de aquello que se está descomponiendo. De la crisis y descomposición se va al momento constitutivo. De ahí se recorre todo el camino que lleva a la crisis como punto de partida de indagación. No hay un modelo teleológico subyacente en fases sucesivas de desarrollo a partir de las cuales se ordenan los hechos históricos (TAPIA en AGUILUZ y DE LOS RÍOS, 2003). En ese sentido,

La crisis, por tanto, es el movimiento de estas sociedades y quizá de las sociedades en general. De aquí se derivan las cuestiones del momento del conocimiento social, es decir, de la súbita capacitación del sujeto, que es la clase para conocer lo que antes estaba vedado, de la presentación "llena" de la sociedad [...] la crisis como escuela, porque sólo la clase que se ha preparado puede en su momento conocer lo que ocurre (ZAVALETA, 1974, p. 21).

Si el obstáculo de una sociedad es su propio desconocimiento ya que "su propio conjunto de determinaciones la hace incapaz de volverse sobre sí misma", su propia capacidad teórica, la autoconciencia sin duda vendrá de la mano de los momentos más álgidos del quiebre del orden establecido.

Y no sólo eso, el problema, el alma del problema es el desconocimiento del sujeto como perteneciente a una clase, más aún, en tanto sujeto que desconoce su potencialidad como sujeto político.

El sujeto que intenta encarar la relación de conocimiento con el objeto es un sujeto que no se asume como sujeto completo, total, no es entonces un sujeto que se reconoce en un mismo tiempo como elemento social, político y productivo. Su visión del lugar está fragmentada, su acto productivo lo aleja de su carácter social y político. La clase entonces no está pre-constituida, no es un elemento dado. No es tal clase social hasta que puede asumirse como sujeto social, político y productor en plenitud, esto es, cuando es capaz de desplegar su praxis entendida como actividad política de transformación (REYNA, 2012, p. 119). 


\section{LOS MOMENTOS CONSTITUTIVOS}

Podrían caracterizarse tres momentos del pensamiento, cristalizados en la obra de Zavaleta. Un primer momento, expreso en la obra "Bolivia: El desarrollo de lo conciencia nacional" de 1967. Un segundo momento, menos nacionalista y en miras a un desarrollo del sujeto proletario en particular, que incorpora y profundiza el análisis del horizonte de visibilidad a partir de la lente marxista, que encuentra en "El poder dual", de 1974, su momento cumbre. Y finalmente, el momento analítico más fecundo que se cristaliza en "Las masas en noviembre" de 1983, y "Lo nacional popular en Bolivia" que se publica posterior a su muerte, en 1986. Esto, sin desconocer la fecundidad de otros ensayos publicados luego de su fallecimiento en obras escogidas.

Lo cierto es que de esta última etapa de creación surge un breve escrito denominado "El Estado en América Latina" (1986) que refiere a las distintas características de los Estados de la región, partiendo de sus independencias, llegando a la pregunta por el origen de una teoría general del Estado en el marco teórico del marxismo y sus condicionantes. En este particular Zavaleta refiere a dos aspectos importantes para profundizar en nuestra problematización guía. La primera es su consideración sobre los momentos constitutivos. Estas instancias a que, más allá de las transformaciones y continuidades del Estado y de la sociedad civil, poseen un grado más alto de profundidad, de confluencia específica, de sedimentación y por lo tanto, de permanencia (ANTEZANA, 1991). Estos momentos permiten reconstruir trayectorias y construir vis a vis una metodología en la cual aspectos marcantes, constituyan la genealogía. La colonización sería entonces un momento constitutivo, así como la conformación de la nación sería otro y la sedimentación del Estado como forma de dominación actual un tercero posible.

En segundo lugar, Zavaleta remite a la indisociabilidad entre estructura y superestructura, acercándose a la perspectiva gramsciana. En ese particular refiere al Estado como constructor o productor de "sustancia estatal". Esta idea condensa la fetichización del aparato del Estado e incorpora la dimensión valorativa inmersa en su propia reproducción.

En rigor, en Zavaleta Mercado, la noción de Estado supone dos usos. Por un lado, tenemos al Estado como algo concreto, objetivo, histórico, el que sería sólo una parte de la totalidad posible, o sea, en sus términos, una 'forma aparente'; por otro lado, tenemos al Estado, en su sentido más estricto, como aquel momento - casi un telos utópico, se diría - donde sucedería 'la disolución del factum estatal en la sociedad civil' (ANTEZANA, 1991, p. 133).

De tal manera que en una formación social abigarrada, se presentan de manera coexistente diversos momentos constitutivos, lo que otorga especificidad a los Estados de condición multisocietal. Eso en parte explica algunas de las dificultades reales que se presentan a la hora de analizar la historia en países con formaciones sociales diversas, encontradas, abigarradas.

Finalmente, cabe recuperar una herramienta analítica más, presente en los estudios de nuestro referente teórico boliviano. Al hacer referencia al desarrollo de sucesos contraofensivos post-revolución del 52 , Zavaleta recurre a un concepto que es reconocible 
en otras instancias políticas latinoamericanas. Hace referencia a la paradoja señorial, en tanto momento de solapamiento de intereses en que los sectores dominantes se apropian de las victorias populares. En sus términos, "en el momento mismo del auge de las masas [...] los pueblos miran a veces como su liberación a lo que suele no ser sino una disputa de reemplazo entre las estirpes de sus amos" (ZAVALETA, 1986, p. 45). Esa noción colabora en explicar las aparentes conquistas de los sectores populares y a su vez, el alcance limitado de las luchas que encuentran en el Estado, un eco vacío. Explica aún más, la pervivencia de intereses de sectores dominantes que por canales institucionalizados, ocupando espacios específicos en el Estado, se reproducen más allá del color del gobierno en el poder. La paradoja no desconsidera el solapamiento de los sectores dominantes en los resultados alcanzados en luchas francamente populares. A modo de dinámica parasitaria, abundan en la región casos en que las luchas populares terminan siendo más útiles a los fines de sectores acomodados, con espacio reservado en sectores de poder, que a los propios sectores que dieron su vida por el cambio social.

\section{PROPUESTA DE CIERRE INACABADO}

El conocimiento que colaboramos en recuperar para poner en debate suele limitarse a tejer relaciones entre procesos que ocurren en la sociedad civil, de manera inicialmente aislada, para luego, en un segundo momento, vincularlo de manera articulada con aquello que sucede en el Estado o en las instituciones políticas hegemónicas. Esa vinculación suele perder de vista la interrelación de ambos espacios en términos de constitución o incluso, inmanencia. En términos gramscianos, esta conjunción, implica considerar a la sociedad como espacio de reproducción cotidiana de ideas y procesos que constituyen al Estado, y que en su vida, lo retroalimentan. Al mismo tiempo, es el Estado la entidad en que los intereses de clase se unifican y se presentan como garantes del interés general.

Por todo esto, entendemos que a partir de las variables conceptuales enumeradas, es posible explicar el alcance de una supremacía hegemónica que se materializa en relaciones sociales incluso críticas. Relaciones basadas mucho más los consensos implícitos que como vasos comunicantes reproducimos, que en estrategias coercitivas.

El propio proceso de dominación moldea las palabras, las imágenes, los símbolos, las formas, las organizaciones, las instituciones y los movimientos utilizados por las poblaciones subalternas para hablar de la dominación, confrontarla, entenderla, acomodarse o resistirse a ella. Lo que la hegemonía construye no es, entonces, una ideología compartida, sino un marco común material y significativo para vivir a través de los órdenes sociales caracterizados por la dominación, hablar de ellos y actuar sobre ellos (ROSEBERRY, 1994, p. 220).

Debido a eso, autores como el mencionado Roseberry consideran que el poder del Estado descansa no tanto en el consentimiento de sus súbditos sino en las formas reguladoras y coercitivos del Estado que crean identidades, como sellos, trámites, pago de impuestos, registros, rutinas o presentación de documentos. La hegemonía construye a todas luces un material común, al actuar y al hablar. Tal vez por estas razones, entre otras, el Estado resulte tan atractivo y por consiguiente tan sorprendentemente resi- 
liente. Tal vez por esta razón, entre otras signadas por los momentos constitutivos de cada realidad nacional, la forma de organización naturalizada sea la alimentada bajo el formato de Estado.

Es posible señalar que, luego del año 2006, se cristalizan perfiles de gobiernos que transforman la acción estatal y en ese sentido, reconfiguran el vínculo con sociedades civiles más activas y revitalizadas. Aun así, el control del estado parece inducir a una búsqueda de representación completa, centralizada, de difícil tolerancia al derrotero de organizaciones políticas autónomas que no buscan en el Estado eco. Como hemos podido observar, en algunos casos, movimientos sociales autónomos signados por la lucha territorial fueron asimilados a la dinámica gobiernista, en otros casos, movimientos políticos de difícil precisión teórico-analítica - en virtud de su parecido con movimientos sociales - fueron impulsados "desde arriba" por los gobiernos progresistas convirtiéndolos en canales de aplicación de políticas públicas. Estos movimientos políticos como La Cámpora en Argentina o las brigadas juveniles del PSUV en Venezuela mixturan su participación en prácticas de gobierno con militancia y acción política comunitaria complejizando el análisis en torno de las fronteras de la movilización social.

La actual era de movilizaciones abre un sinfín de confusiones deliberadas que, dependiendo de la lente con que se mira, puede conducir a lecturas confusas. Y a este punto cabe mencionar también la movilización de sectores conservadores que se apropian de la calle manifestando discursivamente una "voluntad de ampliación democrática" - en reminiscencias memoriales al proceso de transición democrática -, e invocando un espíritu republicano para deslegitimar opciones francamente democráticas. Se proyecta entonces un tipo de movilización con sectores que aparentemente desconocen banderas político-ideológicas - que siempre han sido parte constitutiva de las marchas en tanto repertorio de disputa política no institucional - consagrándose a valores universales como la paz, la libertad y justicia.

Por todo esto, el ciclo de movilizaciones que cierra en 2006 permite entender la bisagra de un Estado que de manera inquebrantable se reposicionó acorde a las distintas circunstancias en la región. Se trata tal vez de una fisura que al menos desde esta perspectiva habilita interpretaciones sobre la potencialidad de las crisis y la posibilidad de construcción de caminos alternativos en escenarios de convulsión y reposicionamiento frente a las formas de organizar el poder.

\section{REFERENCIAS BIBLIOGRÁFICAS}

ABOY CARLÉS, Gerardo. La sangre de Esteno. Transformaciones de la ciudadanía en Argentina: del populismo a la inflexión particularista. En: DELAMATA, Gabriela (Ed.) Movilizaciones sociales: nuevas ciudadanías? Reclamos, derechos, Estado, en Argentina, Bolivia y Brasil. Buenos Aires: Biblos, 2009.

ANTEZANA, Luis. Dos conceptos en la obra de René Zavaleta Mercado: Formación abigarrada y democracia como autodeterminación. En: TAPIA, Luis (Ed.). Pluralismo epistemológico. La Paz: CIDES-UMSA, 1991.

GARCÍA GALLEGOS, Bertha. Singularidad de las Fuerzas Armadas y perfiles militares. De militares a empresarios: la crisis actual de las FFAA. En: MONTES DEL CASTILLO, Ángel. Ecuador contemporâneo. Murcia: Edit.um, 2009. 
GIL QUIROGA, Mauricio. Zavaleta Mercado: ensayo de biografía intelectual. En: ZAVALETA, René. Ensayos, testimonios y re-visiones. Mexico: Flacso-Miño y Dávila, 2006.

MARX, Karl El 18. Brumario de Luis Bonaparte, Madrid: Alianza, 2015 [1852].

ORTEGA REYNA, Jaime. Totalidad, sujeto y política: los aportes de René Zavaleta a la teoría social latinoamericana. En: Revista Andamios, n. 20, v. 9, sept-dic, p. 115-135. México: UACM, 2012.

OSPINA, Pablo. El abril que se llevó al coronel que no murió en el intento. En: Revista Ecuador Debate, n. 65, ago, p. 7-19. Quito: Nueva Sociedad, 2005.

RODAS, Hernán. Religión y cultura. La iglesia ecuatoriana entre la oferta y la demanda religiosa. En: MONTES DEL CASTILLO, Ángel (Ed.). Ecuador contemporáneo: análisis y alternativas actuales. Quito: Edit.um, 2009.

ROSEBERRY, William et al (Eds.) Aspectos cotidianos de la reproducción del Estado, Barcelona: ERA, 1994.

TAPIA, Luis. La producción teórica para pensar América Latina. En: AGUILUZ, Maya y DE LOS RIOS MENDEZ, Norma (Eds). René Zavaleta, ensayo, testimonio y reflexiones. Buenos Aires: UNAM-UNSAM, 2003.

ZAVALETA, René. La revolución democrática de 1952 y las tendencias sociológicas emergentes. En: Ponencia presentada al XI Congreso latinoamericano de Sociología, Costa Rica, 1974.

ZAVALETA, René. Las masas en noviembre. En: ZAVALETA, René (Ed.). Bolivia hoy. Ciudad de Mexico: Siglo XXI, 1983.

ZAVALETA, René. Lo nacional-popular en Bolivia. La Paz: Plural, 2008 [1986].

ZAVALETA, René. Clases sociales y conocimiento. La Paz: Los Amigos del Libro, 1988.

ZAVALETA, René. El Estado en América Latina. La Paz: Los Amigos del Libro, 1989.

ZAVALETA, René. El desarrollo de la conciencia nacional. La Paz: Los amigos del libro, 1990. 